\title{
On the Robustness Properties of M-MRAC
}

\author{
Vahram Stepanyan* \\ Mission Critical Technologies Inc, NASA Ames Research Center, Moffett Field, CA 94035, \\ Kalmanje Krishnakumar ${ }^{\dagger}$ \\ NASA Ames Research Center, Moffett Field, CA 94035
}

\begin{abstract}
The paper presents performance and robustness analysis of the modified reference model MRAC (model reference adaptive control) or M-MRAC in short, which differs from the conventional MRAC systems by feeding back the tracking error to the reference model. The tracking error feedback gain in concert with the adaptation rate provides an additional capability to regulate not only the transient performance of the tracking error, but also the transient performance of the control signal. This differs from the conventional MRAC systems, in which we have only the adaptation rate as a tool to regulate just the transient performance of the tracking error. It is shown that the selection of the feedback gain and the adaptation rate resolves the tradeoff between the robustness and performance in the sense that the increase in the feedback gain improves the behavior of the adaptive control signal, hence improves the systems robustness to time delays (or unmodeled dynamics), while increasing the adaptation rate improves the tracking performance or systems robustness to parametric uncertainties and external disturbances.
\end{abstract}

\section{Introduction}

Robustness analysis of classical linear time invariant (LTI) systems are based on the notions of gain and phase margins, which provide stability guarantee in the presence of unmodeled dynamics and unstructured uncertainties. These stability metrics are computed using linear analysis tools, which cannot be easily extended to inherently nonlinear adaptive system. While the notion of the gain margin for the adaptive systems can be essentially defined in the same way as for the LTI systems, and the estimate of the gain margin can be obtained using the Lyapunov analysis tools (see for example [12]), the notion of phase margin is not defined for the nonlinear systems. On the other hand, time delay margin has been accepted as a viable alternative for the robustness analysis of adaptive systems.

When the adaptation mechanism is turned off, the controller can be viewed as a fixed gain controller, and the adaptive system reduces to an LTI system, when the underlying dynamics is linear. Therefore, it is tempting to freeze the adaptive parameters over a time window, in order to apply the linear analysis tools to estimate the stability margins of the system. However, there is no guarantee that the obtained margins in this fashion relate to the stability of the actual adaptive system. Recently, some methods have been proposed for approximate phase and gain margin analysis of the adaptive systems without terminating the adaptation. In [11], it is proposed to define an LTI system bounding the closed-loop adaptive system then locally evaluate the phase and gain margins for the bounding LTI system. In [14], an LMI based approach is proposed to calculate the stability margins by transforming the nonlinear adaptive system into a linear parameter varying system.

In [9], several linear adaptive control systems in the presence of measurement noise and external disturbances have been compared using the linear analysis tools from the point of view of the performance and robustness.

The time delay margin of the adaptive systems are not readily computable as well. Some methods have been reported in the literature which use Lyapunov based stability tools to estimate it. In [1], the authors

\footnotetext{
*Senior Scientist, Mission Critical Technologies Inc., Senior Member AIAA, vahram.stepanyan@nasa.gov

$\dagger^{\dagger}$ Autonomous Systems and Robotics Tech Area Lead, Intelligent Systems Division, Associate Fellow AIAA, kalmanje.krishnakumar@nasa.gov
} 
use Pade approximation to transform the original time delay system to a higher order system without time delay and approximate the time delay margin. In [7], the Razumikhin method has been use to iteratively estimate the time delay margin. These methods are general to any adaptive control systems, but generate conservative results. In [3] a lower bound for the time delay margin has been obtained that is applicable only to the $\mathcal{L}_{1}$ adaptive control system.

In this paper we do not pursue a goal to estimate the time delay margin of the nonlinear M-MRAC systems, but to show how can one improve it by the proper choice of the design parameters. For this purpose, we follow the Razumikin's approach (see for example $[5,10]$ ) to show that the robustness of MMRAC to time delays can be improved by increasing the error feedback gain, hence the tradeoff between the performance and robustness can be resolved by operating the adaptation rate and the error feedback gain. It is also shown that the M-MRAC design has improved robustness to unmodeled dynamics compared to conventional MRAC design.

In addition to time domain analysis, frequency domain analysis is conducted for the linear M-MRAC systems. Using methods from linear systems theory, several critical transfer functions are computed between the systems input and output signals. These transfer functions, which are called Gang of Six in [2], allow us to compute the stability margins and analyze the performance of the close-loop system in the presence of the measurement noise and external disturbances. By explicitly computing the crossover frequencies and gain and phase margins, it is shown that: 1) the gain margin increases when the error feedback gain is increased and decreases when the adaptation rate is increased; 2) the gain crossover frequency decreases when the feedback gain is increased and increases when the adaptation rate is increased, 3) the phase margin and the time delay margin increase when the feedback gain is increased and decrease when the adaptation rate is increased. On the other hand, the tracking performance and disturbance attenuation properties of the closed loop signal are improved with the increase in the adaptation rate. Hence, the same tradeoff between the performance and robustness is obtained as in the time domain analysis.

The rest of the paper is organized as follows. In Section II we give preliminaries on the M-MRAC design. We provide the robustness analysis to time delays in Section III. The frequency domain analysis of linear M-MRAC systems is given in Section IV. In Section V the robustness of M-MRAC to unmodeled dynamics is provided. Some concluding remarks are given in Section VI.

\section{M-MRAC}

Consider a multi-input multi-output uncertain linear system

$$
\dot{\boldsymbol{x}}(t)=A \boldsymbol{x}(t)+B \boldsymbol{u}(t), \quad \boldsymbol{x}(0)=\boldsymbol{x}_{0},
$$

where $\boldsymbol{x} \in \mathbb{R}^{n}$ and $\boldsymbol{u} \in \mathbb{R}^{q}$ are the state and input of the system respectively, and $A \in \mathbb{R}^{n \times n}$ and $B \in \mathbb{R}^{n \times q}$ are unknown constant matrices. The control objective is to track a reference model

$$
\dot{\boldsymbol{x}}_{r}(t)=A_{m} \boldsymbol{x}_{r}(t)+B_{m} \boldsymbol{r}(t), \quad \boldsymbol{x}_{r}(0)=\boldsymbol{x}_{0},
$$

where $A_{m} \in \mathbb{R}^{n \times n}$ is a Hurwitz matrix and $B_{m} \in \mathbb{R}^{n \times q}$ is of full column rank, assuming that there exists a matrix $K_{1} \in \mathbb{R}^{n \times q}$ and a sign definite matrix $\Lambda \in \mathbb{R}^{q \times q}$ such that the matching conditions hold

$$
\begin{aligned}
B & =B_{m} \Lambda \\
A & =A_{m}-B K_{1}^{\top} .
\end{aligned}
$$

The external command $\boldsymbol{r}(t)$ is usually assumed to be bounded and piece-wise continuous, which has a bounded derivative almost everywhere on $[0, \infty)$. Therefore, it is piecewise uniformly continuous on $[0, \infty)$ in the sense of the definition from [15]. We notice that discontinuous commands such as steps or square waves satisfy these conditions.

The system (1) can be written in the form

$$
\dot{\boldsymbol{x}}(t)=A_{m} \boldsymbol{x}(t)+B_{m} \boldsymbol{r}(t)+B_{m} \Lambda\left[\boldsymbol{u}(t)-\Theta^{\top} \boldsymbol{f}(t)\right],
$$

where $\Theta=\left[\begin{array}{ll}K_{1}^{\top} & K_{2}^{\top}\end{array}\right], \boldsymbol{f}(t)=\left[\begin{array}{ll}\boldsymbol{x}^{\top}(t) & \boldsymbol{r}^{\top}(t)\end{array}\right]^{\top}$ and $K_{2}^{\top}=\Lambda^{-1}$. This suggests that the control signal can be defined as

$$
\boldsymbol{u}(t)=\hat{\Theta}^{\top}(t) \boldsymbol{f}(t),
$$


where $\hat{\Theta}(t)$ is the estimate of the unknown parameter $\Theta$, and is updated online according to the adaptive law

$$
\dot{\hat{\Theta}}(t)=-\gamma \boldsymbol{f}(t) \boldsymbol{e}_{m}^{\top}(t) P B_{m}, \quad \hat{\Theta}(0)=\Theta_{0},
$$

where $\gamma>0$ is the adaptation rate, and $P=P^{\top}>0$ is the solution of the Lyapunov equation

$$
A_{m}^{\top} P+P A_{m}=-Q
$$

for some $Q=Q^{\top}>0$. The error signal $\boldsymbol{e}_{m}(t)=\boldsymbol{x}(t)-\boldsymbol{x}_{m}(t)$ is defined with respect to modified reference model

$$
\dot{\boldsymbol{x}}_{m}(t)=A_{m} \boldsymbol{x}_{m}(t)+B_{m} \boldsymbol{r}(t)+\lambda \boldsymbol{e}_{m}(t), \quad \boldsymbol{x}_{m}(0)=\boldsymbol{x}_{0},
$$

where $\lambda>0$ is a design parameter.

Introducing the parameter estimation error as $\tilde{\Theta}(t)=\hat{\Theta}(t)-\Theta$, the following error dynamics can be derived

$$
\dot{\boldsymbol{e}}_{m}(t)=\left(A_{m}-\lambda \mathbb{I}\right) \boldsymbol{e}_{m}(t)+B_{m} \Lambda \tilde{\Theta}^{\top}(t) \boldsymbol{f}(t) .
$$

It is easy to see that the actual tracking error $\boldsymbol{e}(t)=\boldsymbol{x}(t)-\boldsymbol{x}_{r}(t)$ satisfies the equation

$$
\frac{d}{d t}\left[\boldsymbol{e}_{m}(t)-\boldsymbol{e}(t)\right]=A_{m}\left[\boldsymbol{e}_{m}(t)-\boldsymbol{e}(t)\right]-\lambda \boldsymbol{e}_{m}(t),
$$

since $\boldsymbol{e}_{m}(t)-\boldsymbol{e}(t)=\boldsymbol{x}_{r}(t)-\boldsymbol{x}_{m}(t)$. We notice, that setting $\lambda=0$ results in conventional MRAC design with $\boldsymbol{e}_{m}(t)=\boldsymbol{e}(t)$.

The following theorems give the asymptotic and transient properties of the M-MRAC architecture in the ideal case.

Theorem 1 Let the system (1) be controlled by the M-MRAC scheme given by (5), (8) and (6). Then all closed-loop signals are bounded, and $\boldsymbol{e}(t) \rightarrow 0, \boldsymbol{e}_{m}(t) \rightarrow 0$ and $\tilde{\boldsymbol{u}}(t) \rightarrow 0$ as $t \rightarrow \infty$, where $\tilde{\boldsymbol{u}}(t)=\tilde{\Theta}^{\top}(t) \boldsymbol{f}(t)$.

The theorem is proved using the Lyapunov function

$$
V(t)=\boldsymbol{e}_{m}^{\top}(t) P \boldsymbol{e}_{m}(t)+\frac{1}{\gamma} \operatorname{trace}\left(\Lambda \tilde{\Theta}^{\top}(t) \tilde{\Theta}(t)\right)
$$

and the extended Barabalat's lemma form [15].

Theorem 2 Let the system (1) be controlled by the M-MRAC scheme given by (5), (8) and (6) and

$$
\lambda=\sqrt{2 \alpha \gamma \lambda_{\max }\left(B_{m}^{\top} P B_{m}\right)} .
$$

Then the following bounds hold

$$
\begin{aligned}
\|\tilde{\boldsymbol{u}}(t)\| & \leq \beta_{1} e^{-\nu t}+\beta_{2} \gamma^{-\frac{1}{2}} \\
\|\boldsymbol{e}(t)\| & \leq \beta_{3} e^{-\nu^{*} t}+\beta_{4} \gamma^{-\frac{1}{2}},
\end{aligned}
$$

where $\alpha=\left\|\boldsymbol{x}_{0}\right\|^{2}+\sup _{t}\|\boldsymbol{r}(t)\|^{2}, \beta_{1}, \beta_{2}, \beta_{3}$ and $\beta_{4}$ are positive constants independent of $\gamma, \nu$ is proportional to $\sqrt{\gamma}, \nu^{*}=\min \left[\nu, \nu_{m}\right]$ and $\nu_{m}$ is the rate of decay of $e^{A_{m} t}$.

The proof of the theorem can be found in [13].

\section{Robustness to Time Delays}

In this section we analyze the properties of the M-MRAC design in the presence of a time delay in the input signal. In this case, the system is given by the so called retarded functional differential equation (RFDE), for the analysis of which we use Razumikhin's method. Here, we give some background information on the method. The interested reader can get more detailed information in the monographs [5] and [10]. 


\section{A. Preliminaries on Razumikhin's method}

Let the system be given by a retarded functional differential equation (RFDE) (the terminology and definitions are adopted from [5] and [10])

$$
\dot{\boldsymbol{z}}(t)=\boldsymbol{f}\left(t, \boldsymbol{z}_{t}\right),
$$

where $\boldsymbol{z}_{t}(\theta)=\boldsymbol{z}(t+\theta)$ for $-h \leq \theta \leq 0$ for a given real number $h \geq 0$, and $\boldsymbol{z}_{t_{0}}=\boldsymbol{\phi}$. Here, $\boldsymbol{f}: \mathbb{R} \times C[-h, 0] \rightarrow$ $\mathbb{R}^{n}$ is a continuous functional which is locally Lipschitz in the second argument, $\phi \in C[-h, 0]$, and $C[-h, 0]$ denotes a Banach space of $n$-dimensional real-valued vector-functions with the supremum norm.

Definition 1 ( [5], p. 130) Let $\boldsymbol{f}\left(t, \boldsymbol{z}_{t}\right)=0$ for all $t \in\left[t_{0}, \infty\right)$. The solution $\boldsymbol{z}=0$ of the RFDE (14) is said to be uniformly stable if for any $t_{0}$ and any $\varepsilon>0$ there exists a $\delta=\delta(\varepsilon)>0$ such that $\phi \in \mathcal{B}(0, \delta)$ implies that $\boldsymbol{z}_{t}\left(t_{0}, \boldsymbol{\phi}\right) \in \mathcal{B}(0, \varepsilon)$ for all $t \geq t_{0}$, where $\boldsymbol{z}_{t}\left(t_{0}, \boldsymbol{\phi}\right)$ is the solution of the RFDE (14) through $\left(t_{0}, \boldsymbol{\phi}\right)$, and $\mathcal{B}(0, r)$ denotes a ball of radius $r$ centered at zero.

One way to establish the uniform stability of the trivial solution of RFDE (14) is to check the conditions of the Rasunikhin's theorem (see [5], p. 152)

Theorem 3 Suppose $u, v, w: \mathbb{R}^{+} \rightarrow \mathbb{R}^{+}$are continuous, nondecreasing functions, $u(s), v(s)$ positive for $s>0, u(0)=v(0)=0, v$ strictly increasing. If there is a continuous function $V: \mathbb{R} \times \mathbb{R}^{n} \rightarrow \mathbb{R}$ such that

$$
u(\|\boldsymbol{z}\|) \leq V(t, \boldsymbol{z}) \leq v(\|\boldsymbol{z}\|),
$$

and

$$
\dot{V}(t, \boldsymbol{z}(t)) \leq-w(\|\boldsymbol{z}(t)\|)
$$

if

$$
V(t+\theta, \boldsymbol{z}(t+\theta)) \leq V(t, \boldsymbol{z}(t))
$$

for $\theta \in[-h, 0]$, then the solution $\boldsymbol{z}=0$ of the RFDE (14) is uniformly stable.

As it is shown in [10], the stability of the RFDE (14) can be analyzed using the same Lyapunov function used for the analysis of the ordinary differential equation resulting from RFDE (14) when $h=0$.

\section{B. Nonlinear analysis}

To analyze the robustness properties of M-MRAC system we insert a time delay $t_{d}$ at the input point of the system. That is the control signal is defied as

$$
\boldsymbol{u}(t)= \begin{cases}0, & 0 \leq t \leq t_{d} \\ u\left(t-t_{d}\right), & t>t_{d}\end{cases}
$$

which results in the system

$$
\dot{\boldsymbol{x}}(t)=\left\{\begin{array}{ll}
A \boldsymbol{x}(t), & 0 \leq t \leq t_{d} \\
A \boldsymbol{x}(t)+B u\left(t-t_{d}\right), & t>t_{d}
\end{array},\right.
$$

Obviously, we have

$$
\boldsymbol{x}(t)=e^{A t} \boldsymbol{x}_{0}
$$

on the interval $0 \leq t \leq t_{d}$. The corresponding modified error dynamics take the form

$$
\dot{\boldsymbol{e}}_{m}(t)=\left\{\begin{array}{ll}
\left(A_{m}-\lambda \mathbb{I}\right) \boldsymbol{e}_{m}(t)+\left(A-A_{m}\right) \boldsymbol{x}(t)-B_{m} \boldsymbol{r}(t), & 0 \leq t \leq t_{d} \\
\left(A_{m}-\lambda \mathbb{I}\right) \boldsymbol{e}_{m}(t)+B_{m} \Lambda \tilde{\Theta}^{\top}(t) \boldsymbol{f}(t)-B_{m} \Lambda \int_{t-t_{d}}^{t} \dot{\boldsymbol{u}}(\tau) d \tau, & t>t_{d}
\end{array} .\right.
$$


The error signal $\boldsymbol{e}_{m}(t)$ is readily computed on the delay interval $0 \leq t \leq t_{d}$ to be

$$
\boldsymbol{e}_{m}(t)=\left(A-A_{m}\right) \int_{0}^{t} e^{\left(A_{m}-\lambda I\right)(t-\tau)}\left[e^{A \tau} \boldsymbol{x}_{0}+B_{m} \boldsymbol{r}(\tau)\right] d \tau
$$

The stability of the error system (21) along with the $\dot{\tilde{\Theta}}(t)$ equation readily, which is obtained from the adaptive law (6), is analyzed using the Lyapunov function $V(11)$. First of all, we notice that

$$
\lambda_{\min }(P)\left\|\boldsymbol{e}_{m}\right\|^{2}+\frac{\lambda_{\min }(\Lambda)}{\gamma}\|\tilde{\Theta}\|_{F}^{2} \leq V\left(\boldsymbol{e}_{m}, \tilde{\Theta}\right) \leq \lambda_{\max }(P)\left\|\boldsymbol{e}_{m}\right\|^{2}+\frac{\lambda_{\max }(\Lambda)}{\gamma}\|\tilde{\Theta}\|_{F}^{2},
$$

where the subscript $F$ denotes the Frobenius norm. Obviously the inequality (15) holds with $u(\boldsymbol{z})=$ $\lambda_{\min }(P)\left\|\boldsymbol{e}_{m}\right\|^{2}+\frac{\lambda_{\min }(\Lambda)}{\gamma}\|\tilde{\Theta}\|_{F}^{2}$ and $v(\boldsymbol{z})=\lambda_{\max }(P)\left\|\boldsymbol{e}_{m}\right\|^{2}+\frac{\lambda_{\max }(\Lambda)}{\gamma}\|\tilde{\Theta}\|_{F}^{2}$. Next, we evaluate $\dot{V}$ for $t>t_{d}$. It is straightforward to check that

$$
\dot{V}(t)=-\boldsymbol{e}_{m}^{\top}(t) Q \boldsymbol{e}_{m}(t)-2 \lambda \boldsymbol{e}_{m}^{\top}(t) P \boldsymbol{e}_{m}(t)-2 \boldsymbol{e}_{m}^{\top}(t) P B_{m} \Lambda \int_{t-t_{d}}^{t} \dot{\boldsymbol{u}}(\tau) d \tau
$$

Our goal is to investigate the negativity of $\dot{V}(t)$ depending on the design parameters $\lambda$ and $\gamma$. To this end, we compute $\dot{\boldsymbol{u}}(t)$ using the adaptive laws and the system's dynamics.

$$
\dot{\boldsymbol{u}}(t)=-\gamma B_{m}^{\top} P \boldsymbol{e}_{m}(t)\left[\boldsymbol{x}^{2}(t)+\boldsymbol{r}^{2}(t)\right]+\left[\hat{K}_{1}^{\top} A+B \hat{K}_{1}^{\top}\right] \boldsymbol{x}(t)+\hat{K}_{1}^{\top} B \hat{K}_{2}^{\top} \boldsymbol{r}(t)+\hat{K}_{2}^{\top} \dot{\boldsymbol{r}}(t) .
$$

Therefore $\dot{V}(t)(25)$ can be expressed as

$$
\dot{V}(t)=-\boldsymbol{e}_{m}^{\top}(t) Q \boldsymbol{e}_{m}(t)-2 \lambda \boldsymbol{e}_{m}^{\top}(t) P \boldsymbol{e}_{m}(t)+2 \gamma \boldsymbol{s}_{1}\left(t, t_{d}\right)+\boldsymbol{s}_{2}\left(t, t_{d}\right) .
$$

where we denote $\rho(\tau)=\boldsymbol{x}^{2}(\tau)+\boldsymbol{r}^{2}(\tau)$,

$$
\begin{gathered}
\boldsymbol{s}_{1}\left(t, t_{d}\right)=2 \boldsymbol{e}_{m}^{\top}(t) P B_{m} \Lambda B_{m}^{\top} P \int_{t-t_{d}}^{t} \boldsymbol{e}_{m}(\tau) \rho(\tau) d \tau \\
\boldsymbol{s}_{1}\left(t, t_{d}\right)=2 \boldsymbol{e}_{m}^{\top}(t) P B_{m} \Lambda \int_{t-t_{d}}^{t}\left\{\left[\hat{K}_{1}^{\top}(\tau) A+B \hat{K}_{1}^{\top}(\tau)\right] \boldsymbol{x}(\tau)+\hat{K}_{1}^{\top}(\tau) B \hat{K}_{2}^{\top}(\tau) \boldsymbol{r}(\tau)+\hat{K}_{2}^{\top}(\tau) \dot{\boldsymbol{r}}(\tau)\right\} d \tau
\end{gathered}
$$

The conventional MRAC design corresponds to $\lambda=0$. In this case the design parameters are $Q$ and $\gamma$, assuming that $A_{m}$ and $B_{m}$ are given from the performance specifications. The matrix $Q$ can be chosen from the point of view of maximizing the ratio $\lambda_{\min }(Q) / \lambda_{\max }(P)$, which results in $Q=\mathbb{I}$ (see [8]), p. 342 for details). Hence, $\gamma$ is the only design parameter to be selected from the perspective of minimizing the Lyapunov derivative

$$
\dot{V}(t)=-\boldsymbol{e}_{m}^{\top}(t) \boldsymbol{e}_{m}(t)+2 \gamma \boldsymbol{s}_{1}\left(t, t_{d}\right)+\boldsymbol{s}_{2}\left(t, t_{d}\right),
$$

which can be upper bounded as follows

$$
\dot{V}(t) \leq-\left\|\boldsymbol{e}_{m}(t)\right\|^{2}+2 \gamma t_{d}\left\|P B_{m} \Lambda B_{m}^{\top} P\right\|\left\|\boldsymbol{e}_{t}(\tau)\right\|_{\mathcal{L}_{\infty}}^{2}\left\|\rho_{t}(\tau)\right\|_{\mathcal{L}_{\infty}}+\boldsymbol{s}_{2}\left(t, t_{d}\right)
$$

Here, $\left\|\boldsymbol{e}_{t}(\tau)\right\|_{\mathcal{L}_{\infty}}$ is the norm in the extended $\mathcal{L}_{\infty}$ space [8] (p. 197). Clearly, the upper bound on $\dot{V}(t)$ increases with respect to $\gamma$. In fact, it is increasing in the product $\gamma t_{d}$, which implies that the time delay margin is adversely affected by the large adaptation rate. This is consistent with practical applications and linear analysis (see for example [4] and references therein).

On the other hand, for the M-MRAC design the upper bound (28) has the form

$$
\dot{V}(t) \leq-\left\|\boldsymbol{e}_{m}(t)\right\|^{2}-2 \lambda \lambda_{\min }(P)\left\|\boldsymbol{e}_{m}(t)\right\|^{2}+2 \gamma t_{d}\left\|P B_{m} \Lambda B_{m}^{\top} P\right\|\left\|\boldsymbol{e}_{t}(\tau)\right\|_{\mathcal{L}_{\infty}}^{2}\left\|\rho_{t}(\tau)\right\|_{\mathcal{L}_{\infty}}+\boldsymbol{s}_{2}\left(t, t_{d}\right),
$$

which implies that the upper bound on $\dot{V}(t)$ is a decreasing function in $\lambda$. This observation gives as a capability to increase the time delay margin of M-MRAC systems by the proper choice of $\lambda$. It follows from the Razumikhin's theorem that if for given initial conditions and design parameters $Q=\mathbb{I}, \gamma$ and $\lambda_{1}$ the 
maximum time delay, for which the error system (21) is stable, is $t_{d 1}$, then for some $\lambda_{2}>\lambda_{1}$ the inequality $t_{d 2} \geq t_{d 1}$ holds, where the error system (21) is stable for the time delay $t_{d 2}$.

We test this assertion in a simulation example for a first order system with $A=2, B=1, A_{m}=-1$ and $B_{m}=1$. We choose a unit step at time $t=1 \mathrm{sec}$. as an external command, set $\gamma=1000$ and compute $\lambda$ according to equation (12). Figure 1 displays the performance of conventional MRAC $(\lambda=0)$ and M-MRAC for $x_{0}=0$. It can be noticed that the MRAC performance is not satisfactory even in the ideal case. Next we insert a time delay $t_{d}$ at the input point and numerically compute the system's time delay margin. The conventional MRAC goes unstable at $t_{d}=0.016 \mathrm{sec}$. The time delay margin of the M-MRAC is presented in Table 1 , where $\lambda^{*}=2 \sqrt{\gamma}$, since $x_{0}=0$ and $\sup r^{2}(t)=1$.
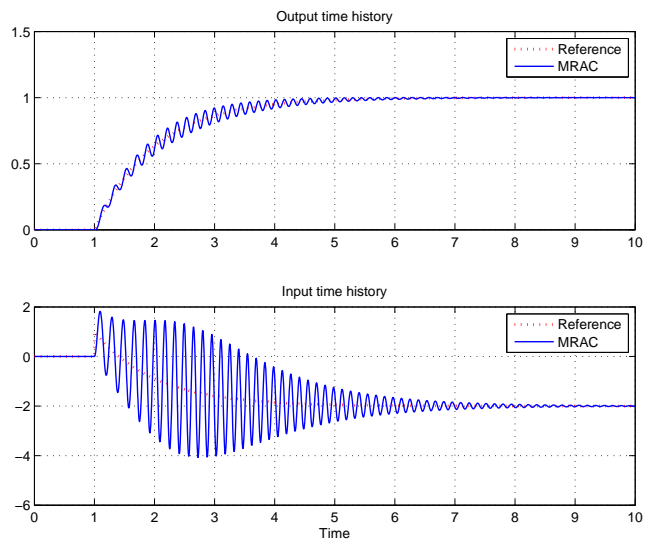

(a) MRAC
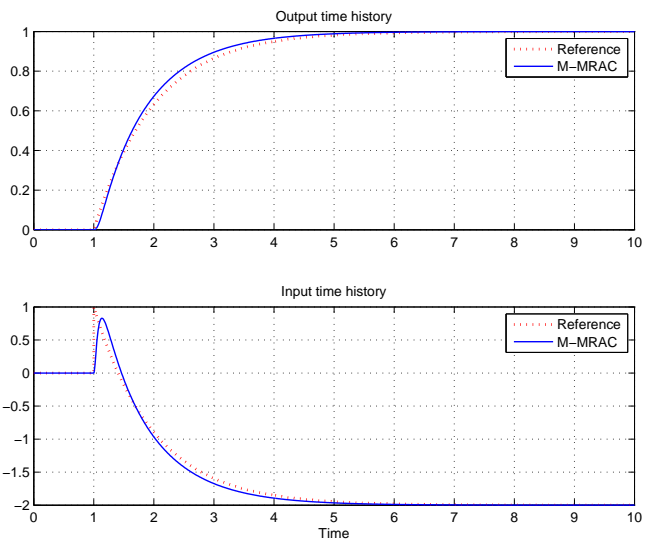

(b) M-MRAC

Figure 1. Input and output time histories in the ideal case.

Table 1. Time delay margin variation with respect to $\lambda$.

\begin{tabular}{|c|c|c|c|}
\hline Error feedback gain $\lambda$ & $\lambda^{*}$ & $2 \lambda^{*}$ & $4 \lambda^{*}$ \\
\hline Time delay margin & 0.039 & 0.80 & 0.126 \\
\hline
\end{tabular}

Obviously the system's robustness to time delays is increased with the increase of $\lambda$, which confirms the analytical predictions. The system's performances corresponding to three cases in Table 1 are displayed in Figure 2. It can be observed that while the control objective is met and the system's output converges to reference model's output for all values of $\lambda$, the transient performance degrades when $\lambda$ is increased from the suboptimal value $\lambda^{*}$. That is $\lambda$ can be used to resolve the tradeoff between the robustness and performance for the given adaptation rate $\gamma$.
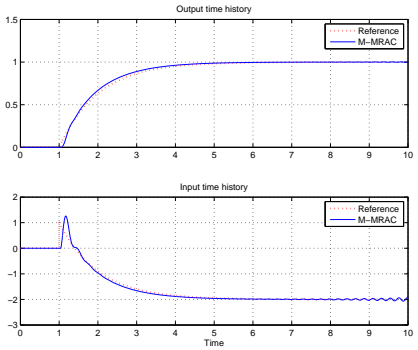

(a) $\lambda=\lambda^{*}, t_{d}=0.039$
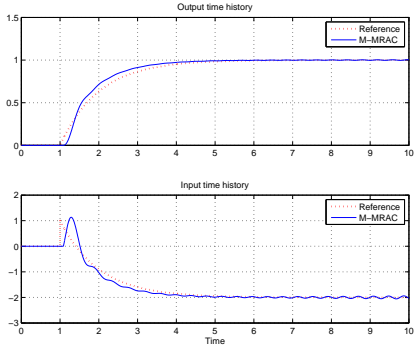

(b) $\lambda=2 \lambda^{*}, t_{d}=0.080$
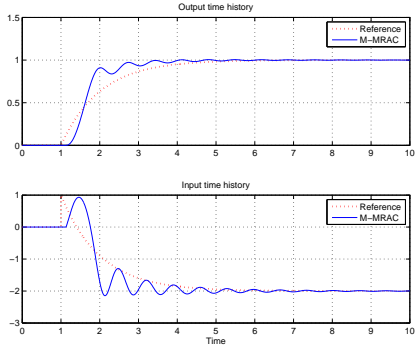

(c) $\lambda=4 \lambda^{*}, t_{d}=0.126$

Figure 2. System's input and output time histories with variable feedback gain and time delays.

It has been noticed that setting the error feedback gain to a time variant quantity $\lambda_{*}(t)=2 \sqrt{\gamma\left[x^{2}(t)+r^{2}(t)\right]}$ improves the both the performance and robustness to time delays of the M-MRAC system. In this case The- 
orem 1 is still valid, hence the above reasonings based on the Razumikin's theorem apply. Table 2 presents the time delay margins of the system with variable $\lambda_{*}(t)$, which are substantially improved over the fixed $\lambda$ case presented in Table 1.

Table 2. Time delay margin variation with respect to time varying $\lambda$.

\begin{tabular}{|c|c|c|c|}
\hline Variable gain $\lambda(t)$ & $\lambda_{*}(t)$ & $2 \lambda_{*}(t)$ & $4 \lambda_{*}(t)$ \\
\hline Time delay margin & 0.055 & 0.104 & 0.162 \\
\hline
\end{tabular}

The corresponding input and output time histories are displayed in Figure 3
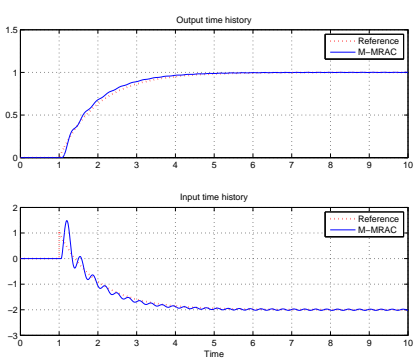

(a) $\lambda=\lambda_{*}(t), t_{d}=0.055$
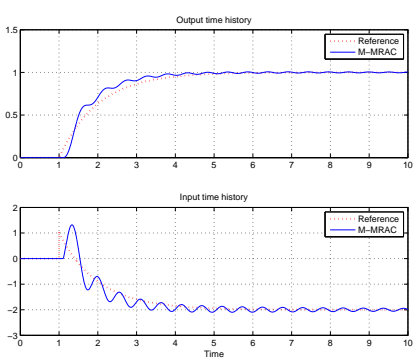

(b) $\lambda=2 \lambda_{*}(t), t_{d}=0.104$
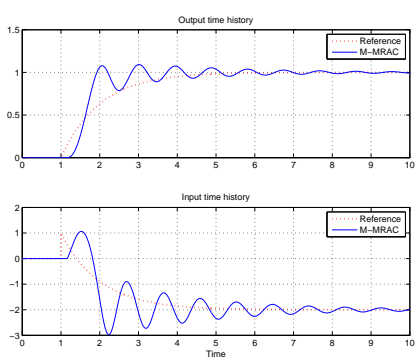

(c) $\lambda=4 \lambda_{*}(t), t_{d}=0.162$

Figure 3. System's input and output time histories with variable feedback gain and time delays.

As in the case of fixed $\lambda$, control objective is met in all cases, but the system's performance degrades with the increase of $\lambda$ at the expense of improved robustness to time delays.

\section{Linear analysis}

When $\mathbf{K}_{1}=0$ and $\mathbf{r}=$ const the M-MRAC design results in the linear time invariant system. In this case, the adaptive control is just an integral control. The system can be written in frequency domain as

$$
\begin{aligned}
\boldsymbol{x}(s) & =\left(s \mathbb{I}-A_{m}\right)^{-1} B \boldsymbol{u}(s) \\
\boldsymbol{x}_{m}(s) & =\left(s \mathbb{I}-A_{m}\right)^{-1} B \boldsymbol{r}(s)+\lambda\left(s \mathbb{I}-A_{m}\right)^{-1}\left[\boldsymbol{x}(s)-\boldsymbol{x}_{m}(s)\right] \\
\boldsymbol{u}(s) & =-B_{m}^{\top} P \frac{\gamma}{s}\left[\boldsymbol{x}(s)-\boldsymbol{x}_{m}(s)\right]
\end{aligned}
$$

and schematically is given in Figure 4. It is easy to see that the controller is of the type of a lead compensator

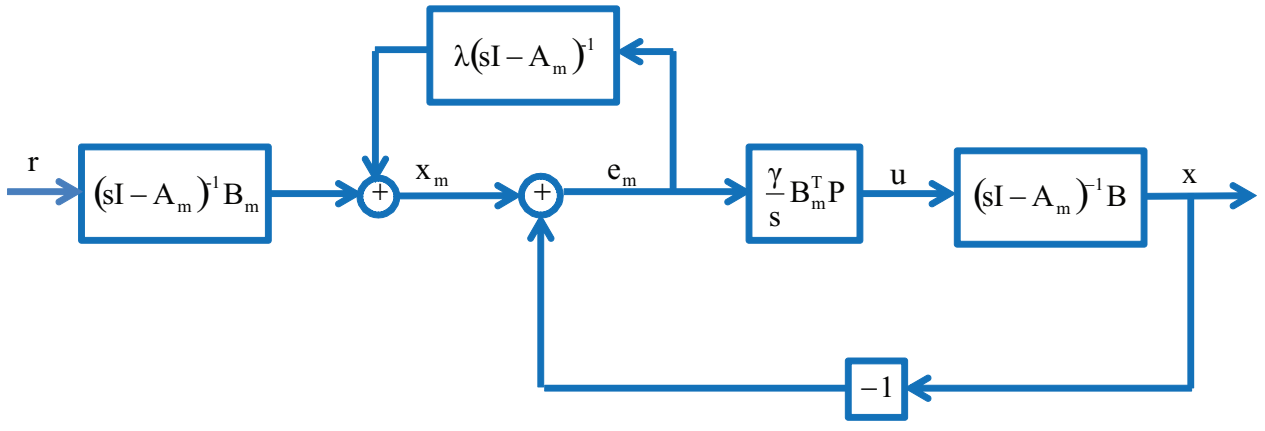

Figure 4. Schematics of the LTI M-MRAC system.

plus integral control, where the lead factor is determined by the parameter $\lambda$. Therefore, $\lambda$ determines the phase margin (or the time delay margin) of the system, whereas $\gamma$ determines the performance. The "sweet" spot given by the formulae (12) defines the relation between the design parameters that minimizes the input and output tracking errors. Increasing $\lambda$ from the "sweet" spot increases the robustness of the system, while 
decreasing it improves the performance. This is in contrast to conventional MRAC design $(\lambda=0)$, where the lead compensator is absent, and the controller is pure integral controller.

To get more analytic insight into the M-MRAC design, we consider a first order system with $A_{m}=-a_{m}$, $B=b>0$ and $b_{m}=a_{m}$. That is

$$
x(s)=\frac{b}{s+a_{m}} u(s), \quad x_{m}=\frac{a_{m}}{s+a_{m}} r(s)+\frac{\lambda}{s+a_{m}}\left[x(s)-x_{m}(s)\right], \quad u(s)=-\frac{\gamma}{s}\left[x(s)-x_{m}(s)\right],
$$

It is easy to see that the loop gain is

$$
L(s)=\frac{b \gamma}{s\left(s+a_{m}+\lambda\right)} .
$$

Therefore, the gain crossover frequency $\omega_{c}$ can be computed from the equation

$$
\left|L\left(j \omega_{g c}\right)\right|=\frac{b \gamma}{\omega_{c} \sqrt{\omega_{c}^{2}+\left(a_{m}+\lambda\right)^{2}}}=1
$$

which results in

$$
\omega_{g c}=\sqrt{\sqrt{\frac{\left(a_{m}+\lambda\right)^{4}}{4}+b^{2} \gamma^{2}}-\frac{\left(a_{m}+\lambda\right)^{2}}{2}},
$$

The phase margin is computed from the equation

$$
P M=\pi-\arg \left(L\left(j \omega_{g c}\right)\right),
$$

which results in

$$
P M=\frac{\pi}{2}-\tan ^{-1}\left(\sqrt{\sqrt{\frac{1}{4}+\frac{b^{2} \gamma^{2}}{\left(a_{m}+\lambda\right)^{4}}}-\frac{1}{2}}\right) .
$$

The corresponding time delay margin is

$$
t_{d}=\frac{P M}{\omega_{g c}}
$$

It can be shown by direct computations that

$$
\frac{\partial \omega_{g c}}{\partial \gamma}>0, \quad \frac{\partial P M}{\partial \gamma}<0, \quad \frac{\partial t_{d}}{\partial \gamma}<0
$$

That is, the phase margin and time delay margin decrease as $\gamma$ is increased for the MRAC and M-MRAC systems, which is consistent with nonlinear analysis. However, in the M-MRAC systems the desired robustness can be achieved with the proper choice of the parameter $\lambda$, which is not available for MRAC systems. It can be shown by direct computations that

$$
\frac{\partial \omega_{g c}}{\partial \lambda}<0, \quad \frac{\partial P M}{\partial \lambda}>0, \quad \frac{\partial t_{d}}{\partial \lambda}>0 .
$$

Therefore, the time delay margin can be increased by increasing $\lambda$. In fact, the desired time delay margin $t_{d}$ can be maintained by setting $\lambda$ according to the equation

$$
\cot \left(t_{d} \sqrt{\sqrt{\frac{\left(a_{m}+\lambda\right)^{4}}{4}+b^{2} \gamma^{2}}-\frac{\left(a_{m}+\lambda\right)^{2}}{2}}\right)=\sqrt{\sqrt{\frac{1}{4}+\frac{b^{2} \gamma^{2}}{\left(a_{m}+\lambda\right)^{4}}}-\frac{1}{2}} .
$$

Figures 5 show the Bode plots for $L(s)$ for $a=2, b=1, \gamma=10000$ in the $\lambda=0$ case, which corresponds to the MRAC design, and in $\lambda=2 \sqrt{b \gamma}-a_{m}$ case, which corresponds to the M-MRAC design. It can be observed that whereas the MRAC design has a phase margin of 1.15 degrees at gain crossover frequency of $100 \mathrm{rad} / \mathrm{sec}$, the M-MRAC design has a phase margin of 76.6 degrees at gain crossover frequency of 48.2 $\mathrm{rad} / \mathrm{sec}$.

Figure 6 shows the behavior of $\omega_{g c}, P M$ and $t_{d}$ with respect to $\lambda$ for $\gamma=10000$, when $\lambda$ is increased proportional to $\sqrt{\gamma}$. The decrease of $\omega_{g c}$, and increase of $P M$ and $t_{d}$ can be observed.

We also provide the Bode plots of the closed loop system, to show that unlike the MRAC system, the M-MRAC does not exhibit any high gain behavior. As it can be seen from Figure 7, MRAC system's gain has a high peak at the frequency $\sqrt{b \gamma}$, but the gain of the M-MRAC system does not exhibit any peak. 


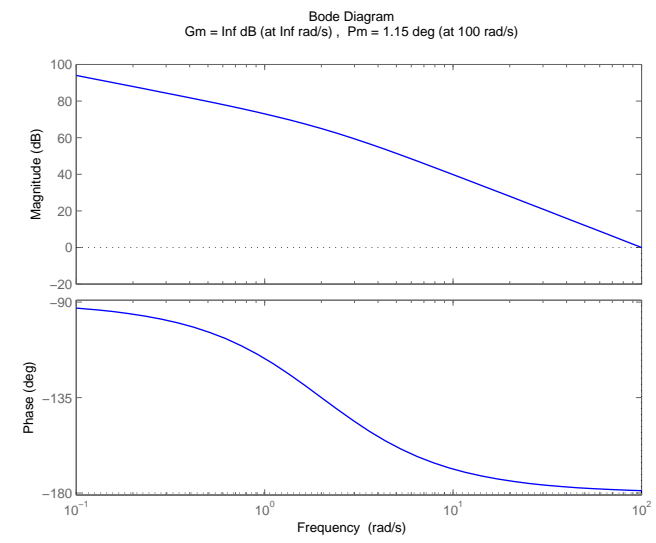

(a) MRAC

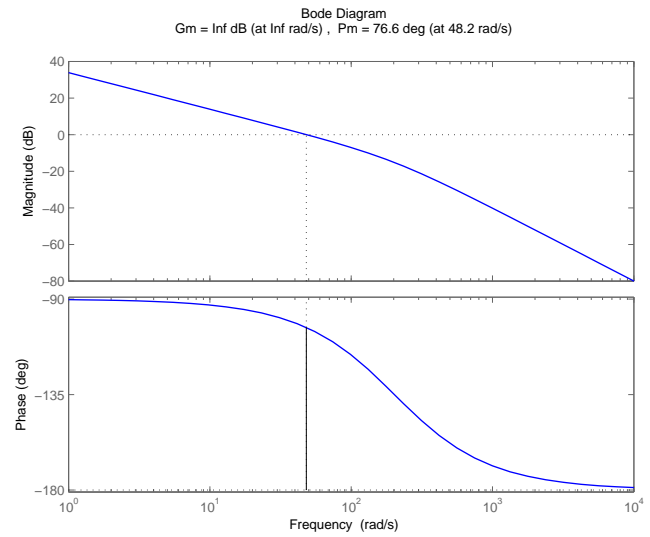

(b) M-MRAC

Figure 5. Bode diagrams with stability margins.

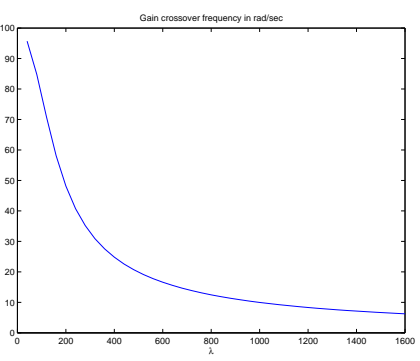

(a) Gain crossover frequency

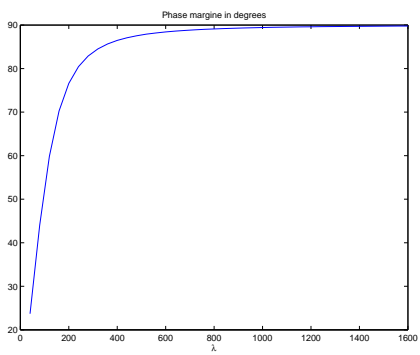

(b) Phase margin

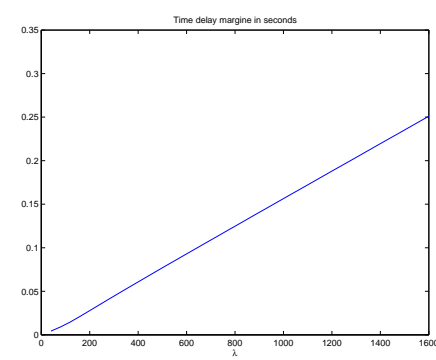

(c) Time delay margin

Figure 6. Robustness properties with respect to $\lambda$.

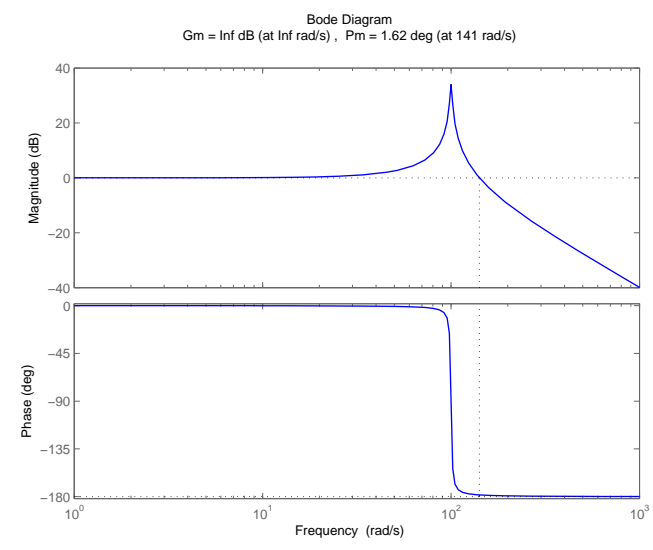

(a) MRAC

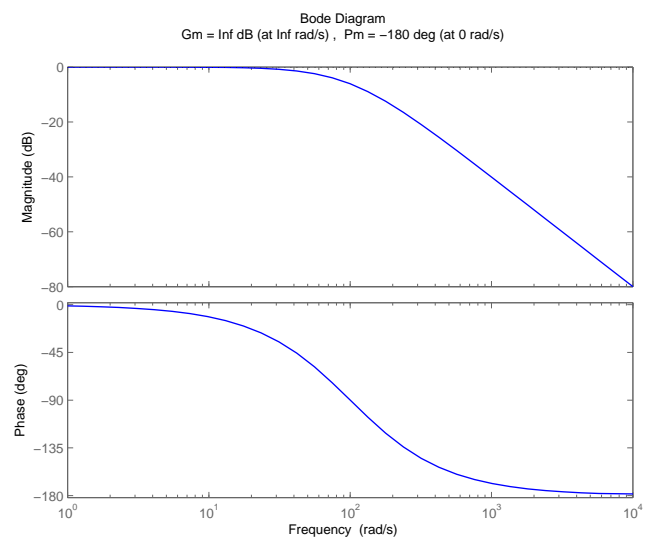

(b) M-MRAC

Figure 7. Bode diagrams of the closed-loop systems.

\section{Frequency Domain Analysis}

In this section we analyze the performance of the closed-loop M-MRAC system (31) in the presence of input and output disturbances. The system is displayed in Figure 8, where $d$ is the input disturbance (load 


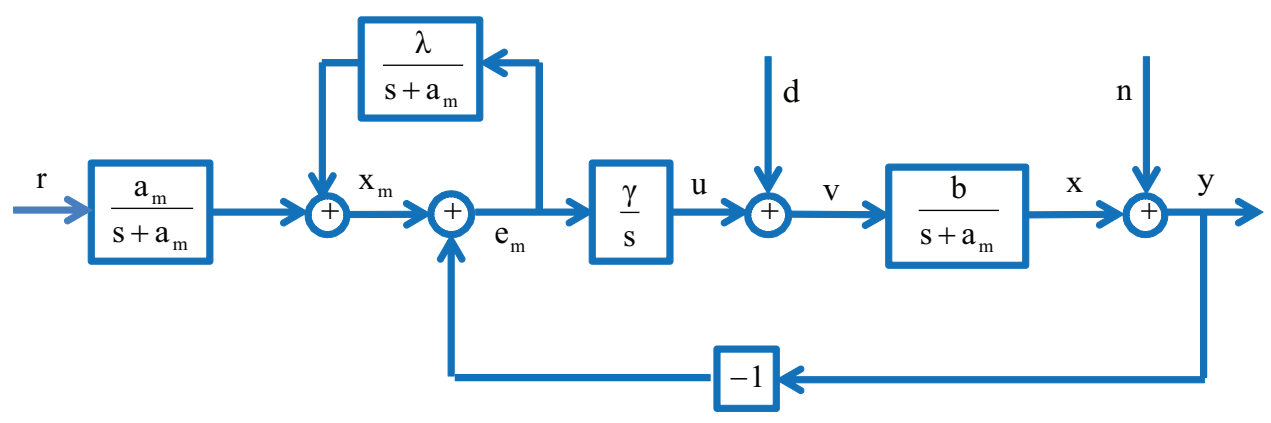

Figure 8. Closed-loop scalar M-MRAC system with input and output disturbances.

disturbance) and $n$ is the output disturbance (measurement noise). It is easy to see that the plant is

$$
P(s)=\frac{b}{s+a_{m}}
$$

the controller is

$$
C(s)=\frac{\gamma\left(s+a_{m}\right)}{s\left(s+a_{m}+\lambda\right)}
$$

and the prefilter (reference model) is

$$
F(s)=\frac{a_{m}}{s+a_{m}} .
$$

Therefore, the following relationships can be obtained from the systems block diagram [2]

$$
\left[\begin{array}{l}
y(s) \\
x(s) \\
v(s) \\
u(s)
\end{array}\right]=\left[\begin{array}{ccc}
\frac{P(s) C(s) F(s)}{1+P(s) C(s)} & \frac{P(s)}{1+P(s) C(s)} & \frac{1}{1+P(s) C(s)} \\
\frac{P(s) C(s) F(s)}{1+P(s) C(s)} & \frac{P(s)}{1+P(s) C(s)} & -\frac{P(s) C(s)}{1+P(s) C(s)} \\
\frac{C(s) F(s)}{1+P(s) C(s)} & \frac{1}{1+P(s) C(s)} & -\frac{C(s)}{1+P(s) C(s)} \\
\frac{C(s) F(s)}{1+P(s) C(s)} & -\frac{P(s) C(s)}{1+P(s) C(s)} & -\frac{C(s)}{1+P(s) C(s)}
\end{array}\right]\left[\begin{array}{c}
r(s) \\
d(s) \\
n(s)
\end{array}\right]
$$

which represent the transfer functions from the inputs $r, d, n$ to the outputs $x, y, u, v$ respectively. The six independent transfer functions involved in the above equation are called the Gang of Six in reference [2]. Their values are given in Table 3 .

Table 3. The Gang of Six for M-MRAC system.

\begin{tabular}{|c|c|}
\hline \hline$G_{y r}(s)=G_{x r}(s)=\frac{b \gamma a_{m}}{\left(s+a_{m}\right)\left(s^{2}+\left(a_{m}+\lambda\right) s+b \gamma\right)}$ & $G_{u r}(s)=G_{v r}(s)=\frac{\gamma\left(s+a_{m}+\lambda\right)}{s^{2}+\left(a_{m}+\lambda\right) s+b \gamma}$ \\
\hline$G_{y d}(s)=G_{x d}(s)=\frac{\left.s+a_{m}+\lambda\right)}{\left(s+a_{m}\right)\left(s^{2}+\left(a_{m}+\lambda\right) s+b \gamma\right)}$ & $G_{y n}(s)=G_{v d}(s)=\frac{\frac{s}{s^{2}+\left(a_{m}+\lambda\right) s+b \gamma}}{G^{2}}$ \\
\hline$G_{x n}(s)=G_{u d}(s)=-\frac{\gamma}{s^{2}+\left(a_{m}+\lambda\right) s+b \gamma}$ & $G_{u n}(s)=G_{v n}(s)=-\frac{\gamma\left(s+a_{m}\right)}{s^{2}+\left(a_{m}+\lambda\right) s+b \gamma}$ \\
\hline
\end{tabular}

First of all we notice that Theorem 2 gives the suboptimal value for $\lambda$ with respect to $\gamma$ according to equation (12). However, for this linear case we can select the optimal value for $\lambda$ as

$$
\lambda_{0}=2 \zeta_{0} \sqrt{b \gamma}-a_{m},
$$

for the damping ratio $\zeta_{0}=1 / \sqrt{2}$. In this case the phase margin (34) reduces to

$$
P M=\frac{\pi}{2}-\tan ^{-1}\left(\sqrt{\sqrt{\frac{1}{4}+\frac{1}{16 \zeta_{0}^{4}}}-\frac{1}{2}}\right),
$$

which is independent of $\lambda$ and $\gamma$ and is equal to $P M=65.5$, which is slightly lower than in the case of suboptimal $\lambda$, as given in Figure 5. On the other hand, the gain crossover frequency depends on $\gamma$ and is 
expressed as

$$
\omega_{g c}=\sqrt{b \gamma} \sqrt{\sqrt{\frac{1}{4}+\frac{1}{16 \zeta_{0}^{4}}}-\frac{1}{2}}
$$

For $\gamma=10000, \omega_{g c}=64.4 \mathrm{rad} / \mathrm{sec}$, which is slightly higher than in suboptimal case. The reason is that in the suboptimal the damping ratio is one.

To check the control objective we apply the final value theorem to the transfer function from the disturbance input $d(s)$ to output $x(s)$

$$
e_{s s}=\lim _{s \rightarrow 0} G_{x d}(s)=\lim _{s \rightarrow 0} \frac{s\left(s+a_{m}+\lambda\right)}{\left(s+a_{m}\right)\left(s^{2}+\left(a_{m}+\lambda\right) s+b \gamma\right)}=0,
$$

where $e_{s s}$ is the steady state error due to a constant disturbance. If we select $\lambda=\lambda_{0}$, for large values of $\gamma$ (fast adaptation)

$$
\frac{b \gamma}{\left(s^{2}+\left(a_{m}+\lambda\right) s+b \gamma\right)} \approx 1
$$

and the closed-loop transfer function $G_{x r}(s)$ approximates the transfer function of the reference model $F(s)$. Moreover, the transfer function from the command $r$ to control $u$ is approximately equal to

$$
\frac{a_{m} \gamma}{\left(s^{2}+\left(a_{m}+\lambda\right) s+b \gamma\right)} \approx \frac{a_{m}}{b}
$$

Body Diagram of $G_{y r}(s)$ and $G_{x r}(s)$

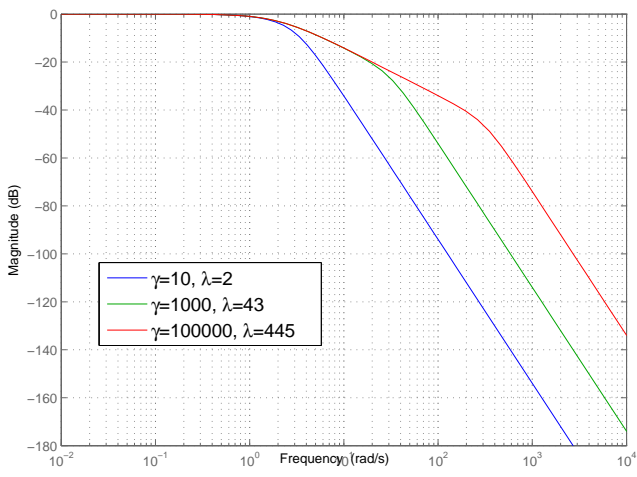

(a) $G_{y r}(s), G_{x r}(s)$

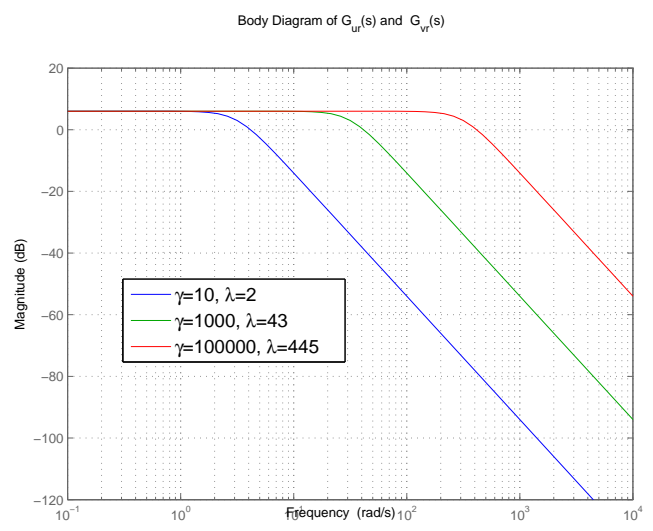

(b) $G_{u r}(s), G_{v r}(s)$

Figure 9. Bode diagrams for the linear M-MRAC system with the optimal value of $\lambda$.

This also can be observed from Figure 9, which shows the Bode magnitude plots for the transfer function $G_{y r}(s), G_{u r}(s)$ for different adaptation rates. Moreover, it can be seen that there is no high gain phenomena for large values of $\gamma$.

To analyze the robustness of M-MRAC to input and output disturbances we plot the Bode magnitude for the remaining members of Gang of Six for different values of the adaptation rate, when the feedback gain $\lambda$ is computed according to equation (36).

It can be observed from Figure 10 that all transfer functions are well behaved with the increase of the adaptation rate except for the noise sensitivity function $G_{u n}(s)$. As Figure $10(\mathrm{~d})$ shows, the peak magnitude of the noise sensitivity function grows with $\gamma$ at higher frequencies. Therefore, the measurement noise will be amplified in the control channel. This phenomenon is similar to one reported in [9] for the conventional MRAC with $\sigma$-modification. However, MRAC with $\sigma$-modification exhibits a nonzero steady state error, thus failing to meet the control objective.

Next, we fix $\gamma$ and vary feedback gain $\lambda$. Figure 11 presents the Body magnitude plots for the members of the Gang of Six for $\gamma=10000$ and $\lambda$ increased from the optimal value $\lambda_{0}$ (36) by order of two. It can be observed that increasing $\lambda$ decreases the peak values of the noise sensitivity function, while the rest of the 


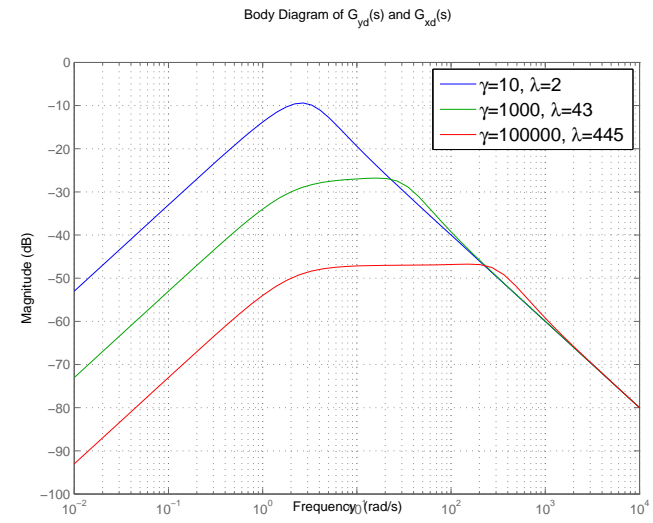

(a) $G_{y d}(s), G_{x d}(s)$

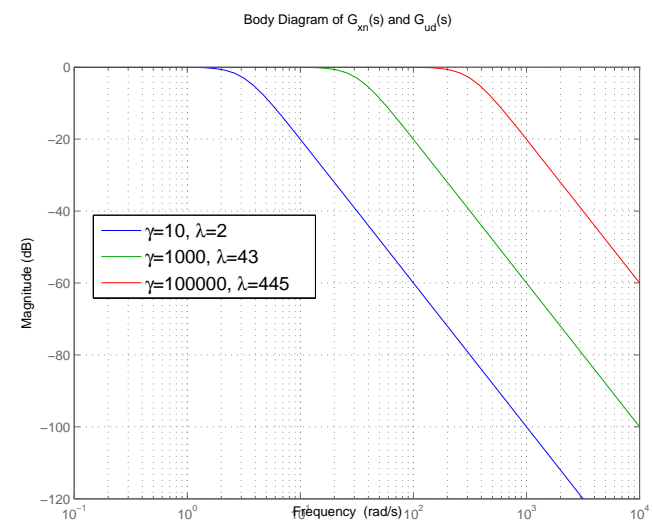

(c) $G_{x n}(s), G_{u d}(s)$

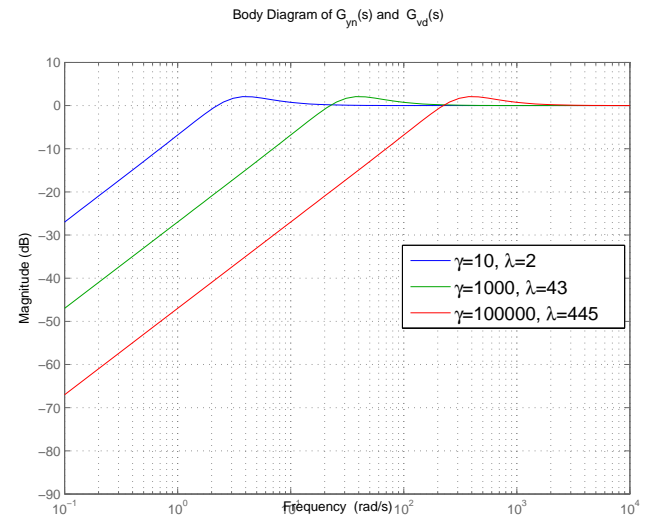

(b) $G_{y n}(s), G_{v d}(s)$

Body Diagram of $G_{u n}(s)$ and $G_{u n}(s)$

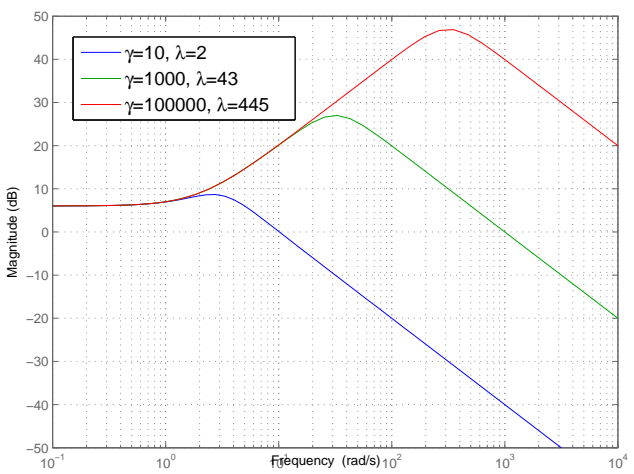

(d) $G_{u n}(s), G_{v n}(s)$

Figure 10. Bode diagrams for the linear M-MRAC system for different adaptation rates.

transfer functions do not experience significant changes. Hence, the robustness to measurement noise can be increased as desired by the proper choice of $\lambda$. Meanwhile, the transfer functions from the command $r(s)$ to the output $y(s)$ and control signal $u(s)$ remain in the acceptable range as it can be concluded from Figure 12 .

\section{Robustness to Unmodeled Dynamics}

In this section we analyze the robustness of M-MRAC to unmodeled dynamics for an example of a first order system. The example is taken from reference [6] (p. 549). The system has a form

$$
y(s)=\frac{b}{s+a}(1+\Delta(s)) u(s),
$$

where $a>0$ is a known constant, $b>0$ is an unknown constant parameter, $\Delta(s)=-\frac{2 \mu s}{\mu s+1}$ is the transfer function of the unmodeled dynamics, and $\mu>0$ is a small parameter. The objective is to track the output of the reference model

$$
y_{r}(s)=\frac{a}{s+a} r(s)
$$




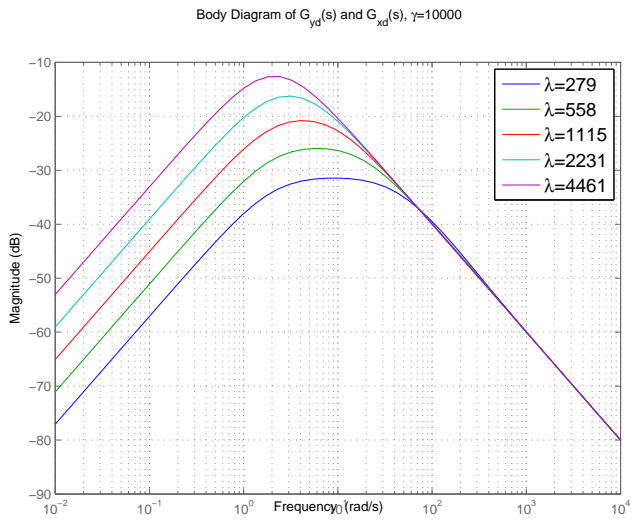

(a) $G_{y d}(s), G_{x d}(s)$

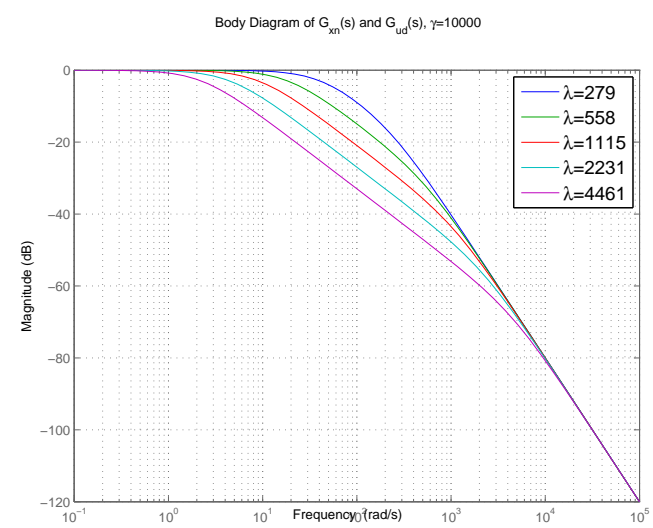

(c) $G_{x n}(s), G_{u d}(s)$

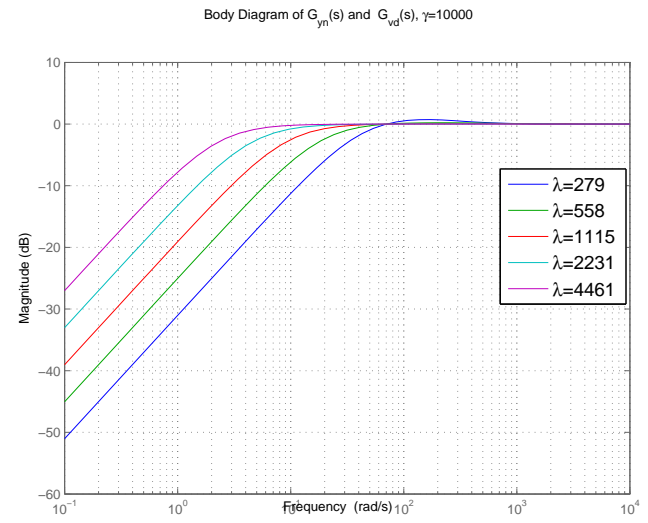

(b) $G_{y n}(s), G_{v d}(s)$

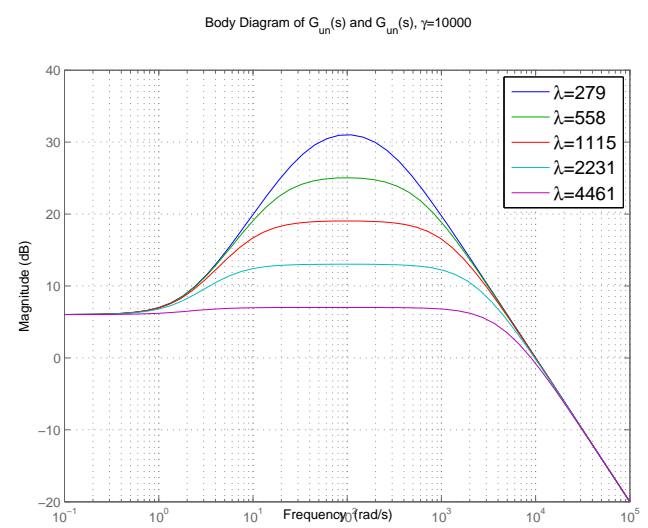

(d) $G_{u n}(s), G_{v n}(s)$

Figure 11. Bode diagrams for the linear M-MRAC system for different values of $\lambda$.

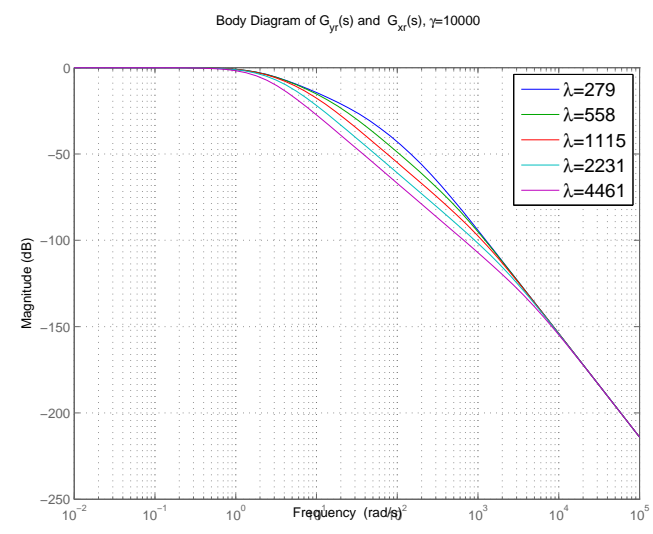

(a) $G_{y r}(s), G_{x r}(s)$

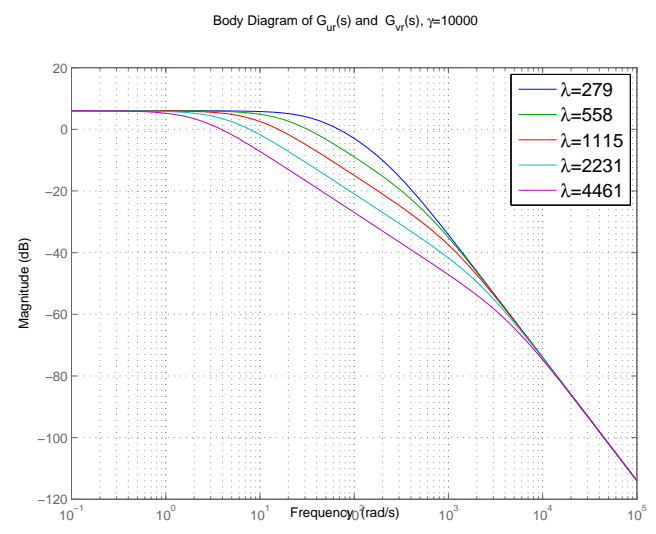

(b) $G_{u r}(s), G_{v r}(s)$

Figure 12. Bode diagrams for the linear M-MRAC system with different values of $\lambda$.

where $r$ is bounded and piecewise uniformly continuous external command. The M-MRAC is designed for the reduced system (that is ignoring the unmodeled dynamics) in the form

$$
\begin{aligned}
y_{m}(s) & =\frac{a}{s+a} r(s)+\frac{\lambda}{s+a}\left[y(s)-y_{m}(s)\right] \\
\dot{\theta}(t) & =-\gamma\left[y(t)-y_{m}(t)\right] r(t) \\
u(t) & =\theta(t) r(t),
\end{aligned}
$$


and is applied to the actual system, which results in the following error dynamics

$$
\begin{aligned}
\dot{e}(t) & =-(a+\lambda) e(t)+b z(t)-b \theta(t) r(t)-a r(t) \\
\dot{z}(t) & =-\frac{1}{\mu} z(t)-\frac{2}{\mu} \theta(t) r(t) \\
\dot{\theta}(t) & =-\gamma e(t) r(t) .
\end{aligned}
$$

If $r=$ constant, the system (42) is LTI with the matrix

$$
\left[\begin{array}{ccc}
-a-\lambda & b & -b r \\
0 & -\frac{1}{\mu} & \frac{2 r}{\mu} \\
-\gamma r & 0 & 0
\end{array}\right]
$$

the characteristic equation of which has the form

$$
s^{3}+\left(a+\lambda+\frac{1}{\mu}\right) s+\left(\frac{a+\lambda}{\mu}-b \gamma r^{2}\right) s+\frac{b \gamma r^{2}}{\mu}=0 .
$$

Using the Routh-Hurwitz criterion on can show that the stability of the system depends on the sign of the third term of the first column of Routh table or equivalently on the sign of

$$
h=(a+\lambda)^{2}+\left(\frac{1}{\mu}-b \mu \gamma r^{2}\right)(a+\lambda)-2 b \gamma r^{2}
$$

When $\lambda=0$ (conventional MRAC), one has $h<0$ whenever

$$
\gamma r^{2}>\frac{1}{\mu} \frac{a+a^{2} \mu}{2 b+\mu a b}
$$

hence the characteristic equation has two roots in the open right-hand side $s$-plane, which coincides with the result derived in [6] in the case of $a=1$ and $b$ replaced with $2 b-1$. That is, a fast adaptation in the conventional MRAC design leads to instability in the presence of the unmodeled dynamics $\Delta(s)$. Unlike MRAC design, M-MRAC design is robust to considered unmodeled dynamics, because we can make $h>0$ by setting

$$
\lambda+a>\frac{1}{2}\left(b \mu \gamma r^{2}-\frac{1}{\mu}\right)+\sqrt{\frac{1}{2}\left(b \mu \gamma r^{2}-\frac{1}{\mu}\right)^{2}+2 b \gamma r^{2}} .
$$

In this case all roots of the characteristic equation are in open left-hand-side $s$-plane, hence the system (42) is exponentially stable, no mater how big $\gamma$ is.

Figure 13 displays the M-MRAC performance from zero initial condition for $a=1, b=2, r=1$ and $\gamma=10000$ (fast adaptation). $\lambda$ is set equal to the right hand side of the inequality (43), where $b$ is replaced with a known upper bound $b_{0}=5$. It can be observed from Figure 13 (a) that in addition to asymptotic tracking, system's both output and input signals have good transient response in the presence of unmodeled dynamics with $\mu=0.1$. When the unmodeled dynamics are more significant $(\mu=0.5)$, the M-MRAC still meets the control objective, but the transient behavior degrades, as it can be viewed in Figure 13 (b).

\section{Conclusions}

We have presented robustness analysis of M-MRAC systems to time delays. The Razumiknin's method was adopted to show that the time delay margin of the M-MRAC systems can be improved by increasing the error feedback gain, whereas the large adaptation rate improves the tracking performance. This tradeoff has been confirmed analytically via the frequency domain analysis in the case of linear adaptive systems. In the linear case the robustness and performance analysis have been also provided in the presence of load disturbances and measurement noise. The robustness of the M-MRAC design to unmodeled dynamics is analyzed for a first order system in comparison with the conventional MRAC system.

The future research will address the robustness of M-MRAC design to measurement noise and unmodeled dynamics in general case of adaptive systems. 

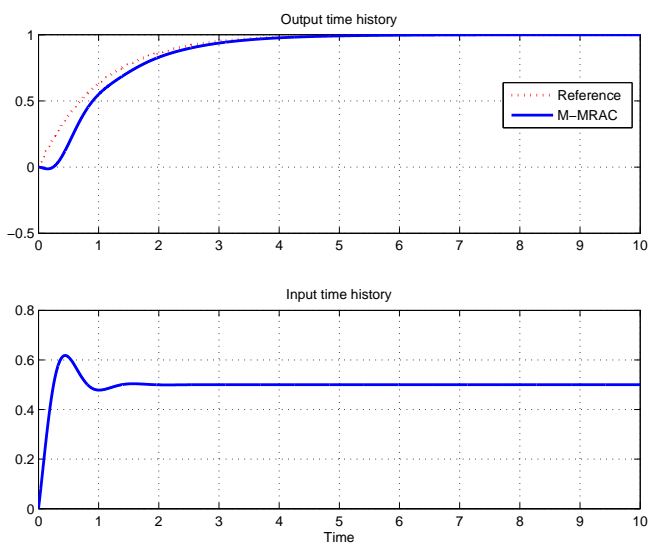

(a) $\mu=0.1$
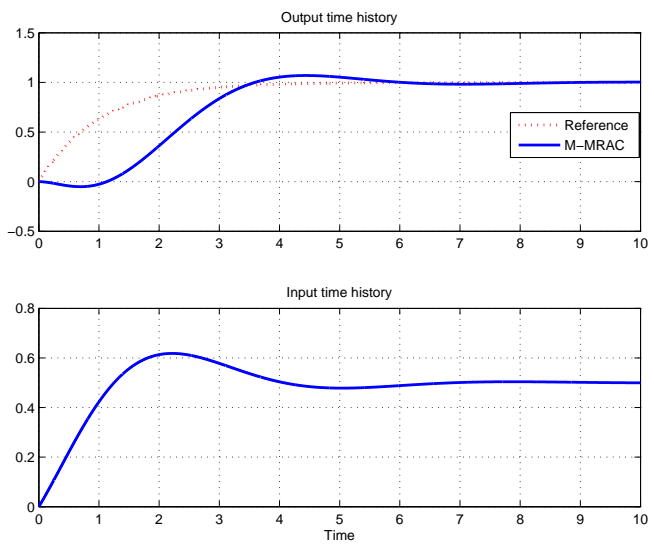

(b) $\mu=0.5$

Figure 13. Bode diagrams for the linear M-MRAC system with the optimal value of $\lambda$.

\section{References}

${ }^{1}$ A. Annaswamy, J. Jang, and E. Lavretsky. Stability Margins for Adaptive Controllers in the Presence of Time-Delay. In Proc. of the AIAA Guidance, Navigation, and Control Conference,AIAA 2008-6659, 2008.

${ }^{2}$ K. J. Astrom and R. M. Murray. Feedback Systems. Princton University Press, Prinston, NJ, 2008.

${ }^{3}$ C. Cao and N. Hovakimyan. Stability Margins of $\mathcal{L}_{1}$ Adaptive Control Architecture. IEEE Transactions on Automatic Control, 55(2):480-487, 2010.

${ }^{4}$ C. Cao, V. V. Patel, C. K. Reddy, and N. Hovakimyan. Are Phase and Time-Delay Margins Always Adversely Affected by High Gain? In Proceedings of the AIAA Guidance, Navigation and Control Conference, Keystone, CO, August 2006. 1993.

${ }^{5}$ J. K. Hale and S. M. Verduyn Lunel. Introduction to Functional Differential Equations. Springer-Verlag, New York, NY,

${ }^{6}$ P. A. Ioannou and J. Sun. Robust Adaptive Control. Prentice Hall, New Jersey, 1996.

${ }^{7}$ A. K. Ishihara, S. Ben-Menahem, and N. T. Nguyen. Time Delay Margin Computation via the Razumikhin Method for an Adaptive Control System. In Proceedings of the AIAA Guidance, Navigation and Control Conference, Chicago, IL, August 2009 .

${ }^{8}$ H.K. Khalil. Nonlinear Systems, Third Edition. Prentice Hall, New Jersey, 2002.

${ }^{9}$ E. Kharisov, N. Hovakimyan, and K. J. Astrom. Comparison of Several Adaptive Controllers According to Their Robustness Metrics. In Proceedings of the AIAA Guidance, Navigation and Control Conference, Toronto, Canada, August 2010.

${ }^{10} \mathrm{~V}$. Kolmanovskii and A. Myshkis. Introduction to Theory and Application of Functional Differential Equations. Kluwer Academic Press, Dordrecht, The Netherlands, 1999.

${ }^{11}$ N. Nguyen and K. Krishnakumar. Hybrid Intelligent Flight Control with Adaptive Learning Parameter Estimation. Journal of Aerospace Computing, Information, and Communication, 6:171-186, March 2009.

${ }^{12}$ Q. Sang and G. Tao. Gain Margins of Model Reference Adaptive Control Systems. In Proc. of the 7th World Congress on Intelligent Control and Automation, Chongqing, China, June 2008.

${ }^{13} \mathrm{~V}$. Stepanyan and K. Krishnakumar. M-MRAC for Nonlinear Systems with Bounded Disturbances. In Proc. of the IEEE Conference on Decision and Control, Orlando, FL, 2011.

${ }^{14}$ B.-J. Yang, T. Yucelen, A. J. Calise, and J.-Y. Shin. An LMI Based Analysis of Stability Margins in Adaptive Flight Control. In Proceedings of the AIAA Guidance, Navigation and Control Conference, Chicago, IL, August 2009.

${ }^{15}$ F. Zhang and N. E. Leonard. Coordinated Patterns of Unit Speed Particles on a Closed Curve. Systems and Control Letters, 56:397 - 407, 2007. 